\title{
Upper and lower respiratory tract microbiota in horses: bacterial communities associated with health and mild asthma (inflammatory airway disease) and effects of dexamethasone
}

\author{
Stephanie L. Bond ${ }^{1}$, Edouard Timsit ${ }^{* *}$, Matthew Workentine', ${ }^{1}$ Trevor Alexander ${ }^{2}$ and Renaud Léguillette ${ }^{1 *}$ (I)
}

\begin{abstract}
Background: The microbial composition of the equine respiratory tract, and differences due to mild equine asthma (also called Inflammatory Airway Disease (IAD)) have not been reported. The primary treatment for control of IAD in horses are corticosteroids. The objectives were to characterize the upper and lower respiratory tract microbiota associated with respiratory health and IAD, and to investigate the effects of dexamethasone on these bacterial communities using high throughput sequencing.

Results: The respiratory microbiota of horses was dominated by four major phyla, Proteobacteria (43.85\%), Actinobacteria (21.63\%), Firmicutes (16.82\%), and Bacteroidetes (13.24\%). Fifty genera had a relative abundance $>0.1 \%$, with Sphingomonas and Pantoea being the most abundant. The upper and lower respiratory tract microbiota differed in healthy horses, with a decrease in richness in the lower airways, and 2 OTUs that differed in abundance. There was a separation between bacterial communities in the lower respiratory tract of healthy and IAD horses; 6 OTUs in the tracheal community had different abundance with disease status, with Streptococcus being increased in IAD horses. Treatment with dexamethasone had an effect on the lower respiratory tract microbiota of both heathy and IAD horses, with 8 OTUs increasing in abundance (including Streptococcus) and 1 OTU decreasing. Conclusions: The lower respiratory tract microbiota differed between healthy and IAD horses. Further research on the role of Streptococcus in IAD is warranted. Dexamethasone treatment affected the lower respiratory tract microbiota, which suggests that control of bacterial overgrowth in IAD horses treated with dexamethasone could be part of the treatment strategy.
\end{abstract}

Keywords: Equine, Metagenomic, 16sRNA, Transtracheal wash, Nasopharyngeal swab, Bronchoalveolar lavage

\section{Background}

Horses can suffer from airway inflammation, resulting in severe or mild asthma [1, 2]. Severe equine asthma (Recurrent Airway Obstruction) is less common than mild asthma in North America, and manifest by increased respiratory effort at rest [1, 3]. Mild equine asthma (also known as Inflammatory Airway Disease [IAD]) affects up to $66 \%$ of the equine population [4]

\footnotetext{
* Correspondence: eftimsit@ucalgary.ca; rleguill@ucalgary.ca

${ }^{1}$ Faculty of Veterinary Medicine, University of Calgary, Calgary, AB, Canada

Full list of author information is available at the end of the article
}

and results in a mild increased resistance to airflow during exercise within the lower airways [5-8]. Clinical signs of IAD are typically subtle at rest, with horses exhibiting normal respiratory effort and occasional coughing; at work, increased nasal discharge, cough and poor performance are observed [8].

Although IAD is primarily an inflammatory process, an infectious component is highly suspected [9-11]. The presence of Streptococcus zooepidemicus, Streptococcus pneumoniae, Actinobacillus spp., and Mycoplasma equihinis in tracheal samples was recently associated 
with IAD, indicating that composition of the lower respiratory tract microbiota could contribute to the pathogenesis [9]. Furthermore, microbial composition and diversity of the bronchial airways in humans with suboptimally controlled asthma has been associated with the degree of bronchial hyperresponsiveness, suggesting that lower airway bacterial communities play a role in asthma pathogenesis [12]. However, as there is limited evidence and controversy on associating IAD with bacterial populations in the trachea, further research is required to confirm this association in horses. Furthermore, there is a need to comprehensively describe the bacterial communities present in health and disease as the overall composition of the bacterial communities, rather than the presence of individual species, is important in defining health and disease [12-14].

Airway inflammation associated with IAD is primarily treated with parenteral corticosteroids (i.e. dexamethasone) [8]. Corticosteroids are effective at controlling airway inflammation and inhibiting airway hypersensitivity and hyperreactivity, thus improving pulmonary function [15]. However, as corticosteroids can cause immunosuppression within the respiratory tract [16], treating IAD with dexamethasone could potentially influence the lower respiratory tract bacterial communities, promoting the overgrowth of specific bacteria, which may in turn contribute to recrudescence of disease upon cessation of treatment. Currently, the impact of dexamethasone on the equine respiratory microbiota is unknown.

The objectives of the current study were therefore (i) to characterize the upper and lower respiratory tract microbiota associated with health and mild IAD (mild asthma) and, (ii) to investigate the effects of dexamethasone on these bacterial communities, using high throughput sequencing.

\section{Methods}

\section{Ethics statement}

This study was conducted in strict accordance with the recommendations of the Canadian Council of Animal Care. The research protocol was reviewed and approved by the University of Calgary Veterinary Sciences Animal Care Committee (AC17-0036).

\section{Animals and study design}

Thirteen deconditioned Thoroughbred horses (geldings) used for chuckwagon racing were studied over a period of 12 days. All horses had a history of coughing, resided on the same property (Okotoks, AB, Canada) and were kept outside in dirt paddocks. They were fed a diet of second-cut alfalfa hay for the duration of the trial, beginning a minimum of 2 days before initial sampling. Nasopharyngeal swabs (NPS), percutaneous transtracheal washes (TTW) and bronchoalveolar lavages (BAL) were performed on all horses $(n=13)$ on day 0 (Fig. 1). Horses were then allocated on day 1 into one of three groups based on their BAL cytology (IAD versus healthy) and random selection (among healthy horses); IAD (horses with mild equine asthma; $n=7$ ), DEX (healthy horses treated with dexamethasone; $n=3$ ) and CONTROL (healthy horses not treated with dexamethasone; $n=3$ ). Horses were considered to have mild equine asthma based on the following inclusion criteria: 1 . a BAL with increased percentage of mast cells $(>3 \%)$ or/ and eosinophils $(>0.5 \%)$ or/and neutrophils $(>10 \%), 2$. absence of laboured breathing at rest [8].

Horses in IAD and DEX groups were then administered dexamethasone (20 mg, IM) every morning for 10 days. No other medications were given to horses for the duration of the trial. On day 11, NPS, TTW and BAL procedures were repeated (Fig. 1).

\section{Sampling procedures}

Horses were pre-medicated with acepromazine maleate (0.07-0.08 mg/kg, IM/IV) approx. Thirty minutes prior to procedures. Horses were sedated to effect with xylazine hydrochloride $(0.4-0.5 \mathrm{mg} / \mathrm{kg}$, IV) and butorphanol tartrate $(0.05-0.1 \mathrm{mg} / \mathrm{kg})$. Following sedation, nasopharyngeal swabs (NPS) were performed first, followed by the percutaneous transtracheal washes (TTW) and then by the bronchoalveolar lavages.

Nasopharyngeal swabs (NPS) were collected as described previously [17], using long guarded swabs $(27 \mathrm{~cm})$ with a rayon bud (Dryswab Veterinary Laryngeal, Medical Wire and Equipment, Corsham, England). Two NPS were obtained per horse (one per nasal cavity). Control swabs $(n=2)$ were collected each sampling day, with the tip of the swab being exposed to the barn air. Immediately after collection, NPS were placed into an Amies transport media $(1.0 \mathrm{~mL})$ and refrigerated at $4{ }^{\circ} \mathrm{C}$. Samples were processed within $10 \mathrm{~h}$ of collection. At processing, each rayon tip was removed from the Amies transport media, which was then transferred into a sterile $1.5 \mathrm{~mL}$ microfuge tube. The Amies and tip were stored in the microfuge tube and original transport container respectively, at $-80{ }^{\circ} \mathrm{C}$ pending DNA extraction.

Percutaneous transtracheal washes were performed as described previously [18]. Briefly, a $10 \mathrm{~cm} x 15 \mathrm{~cm}$ area over the mid-cervical trachea was clipped and surgically prepared. After subcutaneous local anaesthesia (2\% lidocaine, $5 \mathrm{ml}$ ), a stab incision was made through the skin and subcutaneous tissues at the mid-tracheal level and an equine TTW kit was utilized (Mila International, Item number: TW1228) according to manufacturer's instructions. Sterile saline $(15 \mathrm{ml})$ was injected through the catheter, and aspirated immediately. The aspirate was immediately transferred into a sterile $10 \mathrm{~mL}$ plain tube [19] and stored at $4{ }^{\circ} \mathrm{C}$. Controls $(n=2)$ were also 


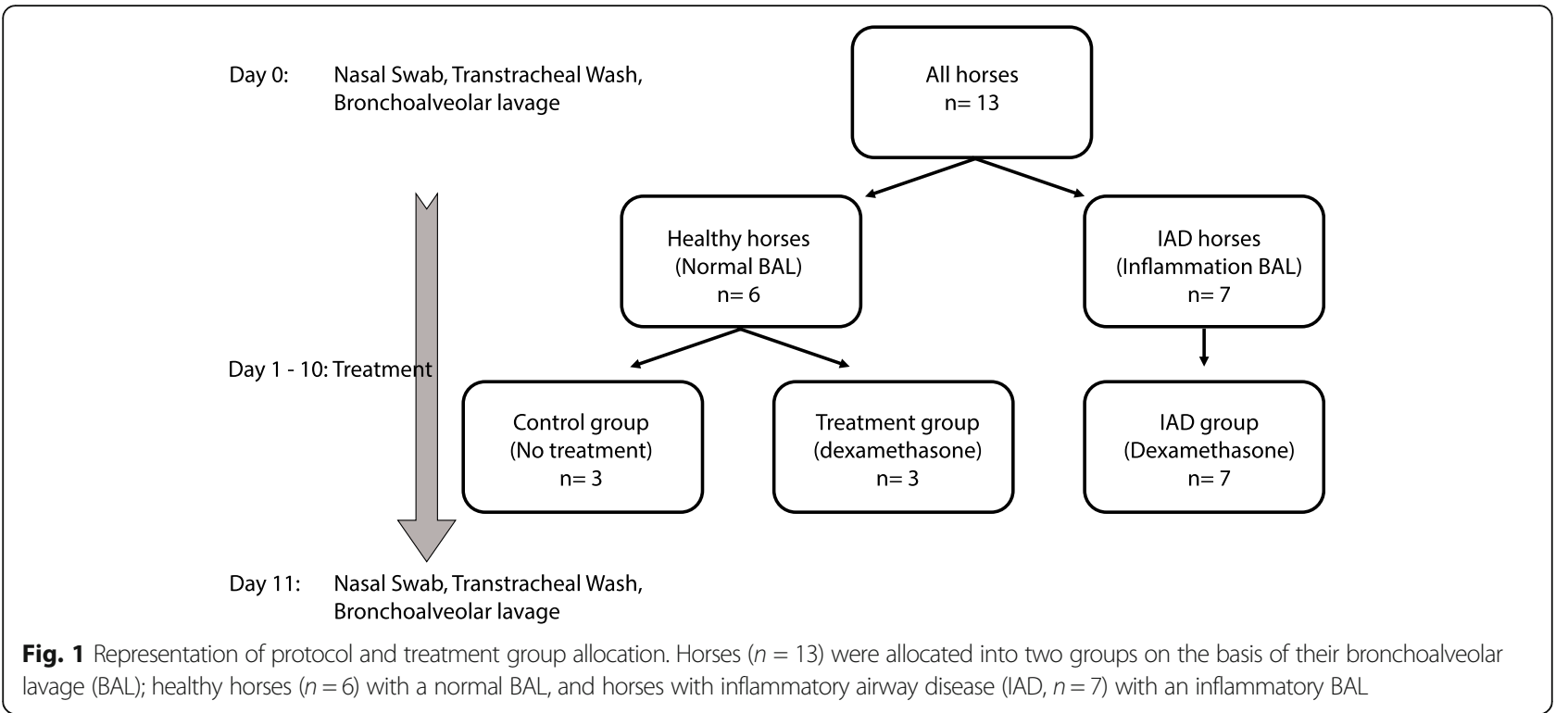

obtained using the same sterile saline, which was flushed into the catheter portion of a sterile TTW kit. Samples were processed within $10 \mathrm{~h}$ of collection. At processing, particulate matter was re-suspended in aspirate through gentle agitation via pipette and $3.0 \mathrm{~mL}$ of aspirate was transferred into 2 sterile $1.5 \mathrm{ml}$ microfuge tubes, which were then centrifuged $(14,000 \mathrm{rpm}, 3 \mathrm{mins})$ to pellet the bacteria. The resulting pellet was stored in the microfuge tubes at $-80^{\circ} \mathrm{C}$ pending DNA extraction.

Bronchoalveolar lavages were performed as described previously [4], with modifications. Briefly, a balloon-tipped BAL tube (Mila International, SKU: BAL300) was inserted into a nostril and progressively passed until its tip was wedged against the wall of a bronchus. During insertion of the BAL tube through the airways, several small boluses of a $0.5 \%$ solution (120 mL total/horse) of lidocaine hydrochloride were administered to desensitize the bronchial mucosa. Two boluses $(250 \mathrm{ml} /$ bolus $)$ of sterile isotonic saline $(0.9 \% \mathrm{NaCl})$ solution were sequentially and rapidly instilled in the bronchus and then immediately aspirated by use of sterile $60 \mathrm{ml}$ syringes. Lavage fluid was recovered and transferred into two $10 \mathrm{~mL}$ sterile EDTA tubes and kept on ice until analysis, which was performed within $6 \mathrm{~h}$ of sample collection. Preparation of slides was performed with $400 \mu \mathrm{l}$ of BAL fluid, which was centrifuged using a Cytospin ( $90 \mathrm{X} \mathrm{g}$ for $5 \mathrm{~min}$ ) to spread the pellet on the slide and stained with modified Wright-Giemsa stain. A differential count was performed on a minimum of 400 cells; epithelial cells were not included in the differential count. A paired nonparametric test (Wilcoxon Signed Rank Test) was used to compare BAL variables between day 0 and 12 . A $p$-value $<0.05$ was considered significant.

\section{DNA extraction}

Total DNA was extracted from NPS and TTW samples using a Qiagen DNEasy Tissue kit (Qiagen Inc., Mississauga, ON, Canada) with the following modifications; briefly, after thawing, the microfuge tubes containing the Amies (NPS samples) and TTW fluid were centrifuged $(13,000 \times$ g for $5 \mathrm{~min})$ again to ensure that bacteria were pelleted before the DNA extraction procedures. The supernatant was discarded. The rayon tip of the NPS was removed from the applicator and then placed in microfuge tube with bacterial pellet for each sample. The pellets were re-suspended in $180 \mu \mathrm{l}$ of enzymatic buffer containing mutanolysin $\left(300 \mathrm{U} \mathrm{ml}^{-1}\right)$ and lysozyme $\left(20 \mathrm{mg} \mathrm{ml}^{-1}\right)$. The mixtures were vortexed and then incubated for 1 hour at $37^{\circ} \mathrm{C}$. Twenty-five $\mu \mathrm{l}$ of proteinase $\mathrm{K}$ and $200 \mu \mathrm{l}$ Buffer AL (without ethanol) were then added, followed by vortexing and incubation at $56{ }^{\circ} \mathrm{C}$ for $30 \mathrm{~min}$. Approximately $300 \mathrm{mg}$ of $0.1 \mathrm{~mm}$ zircon/ silica beads were added and mixed using a Tissue Lyser II (Qiagen) at $30 \mathrm{~Hz}$ for $5 \mathrm{~min}$. The mixtures were then centrifuged $(13,000 \times \mathrm{g}$ for $5 \mathrm{~min})$, and $200 \mu \mathrm{l}$ of ethanol was added to the supernatants, followed by vortexing. The remainder of the protocol of the DNEasy Tissue Kit was followed as per manufacturer instructions. Extracted DNA was stored at $-80{ }^{\circ} \mathrm{C}$ until amplification and sequencing. Blank negative controls (kit only) were included in triplicate during DNA extraction. 


\section{Amplification and sequencing}

The standard Illumina 16S metagenomics library preparation protocol was used (available at: https://support. illumina.com/content/dam/illumina-support/documents/ documentation/chemistry_documentation/16s/16s-meta genomic-library-prep-guide-15044223-b.pdf). All amplification and sequencing steps were carried out at University Core DNA Services, Sequencing and Genetic Analysis Lab (University of Calgary, AB, Canada). The 16S Amplicon PCR forward primer (5'TCGTCGGC AGCGTCAGATGTGTATAAGAGACAGCCTACGGGN GGCWGCAG) and reverse primer (5'GTCTCGTGG GCTCGGAGATGTGTATAAGAGACAGGACTACHVG GGTATCTAATCC) were used to amplify the V3 and V4 regions of the 16S rRNA gene. Illumina sequencing adapters and dual-index barcodes were added to the amplicon target to allow for library pooling prior to sequencing. Briefly, 16S rRNA gene amplicons were generated using a KAPA HiFi HotStart ReadyMix Kit (Kapa Biosystems) with the following PCR conditions: a 3 min initial denaturation at $95^{\circ} \mathrm{C}$ followed by 25 cycles of $95{ }^{\circ} \mathrm{C}$ for $30 \mathrm{~s}, 55^{\circ} \mathrm{C}$ for $30 \mathrm{~s}$, and $72{ }^{\circ} \mathrm{C}$ for $30 \mathrm{~s}$, with a final extension of $5 \mathrm{~min}$ at $72{ }^{\circ} \mathrm{C}$. Amplicon were then purified with Agencourt AMPure XP beads (Beckman Coulter Inc., ON, Canada), and sequenced on an Illumina MiSeq system (Illumina Inc., Victoria, BC, Canada) using the $2 \times 300 \mathrm{bp}$ paired-end sequencing kit. Negative controls were included in triplicate during amplification and sequencing.

\section{Operational taxonomic unit (OTU) table construction}

Raw reads were processed with cutadapt 1.8.3 [20] to remove the primer sequences and any preceding adaptors. Subsequent processing was done using the UPARSE pipeline [21] as implemented in usearch 8.1.1861. The forward and reverse reads were merged using the fastq_mergepairs option of usearch and subsequently filtered with usearch -fastq_filter and an expected error (EE) cut-off of 1 [22] and truncated to maximum length of $420 \mathrm{bp}$. The filtered reads were de-replicated using usearch -derep_fulllength and then clustered at $97 \%$ identity with usearch -cluster_otus and the option '-minsize 2' to remove singleton reads prior to clustering. Taxonomy was assigned to the representative sequences using the RDP naïve Bayesian classifier [23] as implemented in 'assign_taxonomy' function in the $\mathrm{R}$ package dada2 [24] using the RDP training set 14. The final OTU table was constructed with usearch -usearch_global and the options '-strand plus -id 0.97'. OTU sequences were aligned using ssu-align 0.1.1 [25] and a phylogenetic tree built using FastTree 2.1.8. The entire procedure was run as a Snakemake pipeline [26] and code for the pipeline (version 1.0.1) is available on Github (https://github. com/ucvm/vmmp).

\section{Diversity analysis}

Downstream analysis was done in $\mathrm{R}$ 3.3.1 [27] using phyloseq 1.16.2 [28] and vegan 2.4-1 [29]. Mitochondrial and chloroplast sequences were removed as well as the top 20 most abundant OTUs that were present in the negative control samples (Additional file 1: Figure S1). Samples with less than 1000 sequences were also removed from downstream analysis $(n=1)$. Alphadiversity was measured using Chaol and Shannon index [30] (using the whole OTU table) [31]. Differences in alpha-diversity between groups was tested using a Mann-Whitney test, controlling the false discovery rate [32] and using a cut-off of $p<0.05$ for rejecting the null hypothesis of no difference between groups. For $\beta$-diversity, only OTUs with a count of 2 or more in at least $10 \%$ of the samples were retained for further analysis. Between-sample diversity was evaluated using the Bray-Curtis distance metric on proportionally normalized OTU counts and visualized with non-metric multidimensional scaling (NMDS). In this manuscript, we refer to the OTU counts as abundance and the proportionally normalized counts as relative abundance. The generalized linear model framework as implemented in DESeq2 [33] was used to identify OTUs associated with sample type differences (upper versus lower airways), differences between untreated healthy and disease horses, and the effect of dexamethasone treatment. This approach

Table 1 Median (IQR) values for cytologic evaluation of bronchoalveolar fluid obtained before (Day 0) and after 10 days of treatment (Day 11) with intramuscular dexamethasone $(0.05 \mathrm{mg} / \mathrm{kg} \mathrm{SID)}$ for horses with Inflammatory Airway Disease (IAD), and healthy horses (DEX). CONTROL horses were kept in the same environment as both IAD and DEX groups and were sampled at the same time-points, but were given no dexamethasone

\begin{tabular}{|c|c|c|c|c|c|c|}
\hline \multirow[t]{2}{*}{ Variable } & \multicolumn{2}{|l|}{ IAD (7 horses) } & \multicolumn{2}{|l|}{ DEX (3 horses) } & \multicolumn{2}{|c|}{ CONTROL (3 horses) } \\
\hline & Day 0 & Day 11 & Day 0 & Day 11 & Day 0 & Day 11 \\
\hline Neutrophils (\%) & $10(6.2-11.0)$ & $11(6.0-19.7)$ & $3(2.0-4.75)$ & $3(1.7-3.0)$ & $4(2.5-4.0)$ & $2(1.7-3.2)$ \\
\hline Mast cells (\%) & $8(5.2-11.0)$ & $6.5(4.2-7.2)$ & $2(2.0-2.0)$ & $2(1.5-2.2)$ & $2(1.7-2.7)$ & $2(1.5-2.7)$ \\
\hline Eosinophils (\%) & $0(0.0-0.0)$ & $0(0.0-0.0)$ & $0(0.0-0.0)$ & $0(0.0-0.0)$ & $0(0.0-0.2)$ & $0(0.0-0.0)$ \\
\hline Macrophages (\%) & $49(45.5-53.0)$ & $44(37.0-55.5)$ & $62(53.5-64.0)$ & $69(66.5-74.5)$ & $67(57.0-74.2)$ & $62(61.5-63.5)$ \\
\hline Lymphocytes (\%) & $34.5(31.7-36.0)$ & $31(23.2-42.5)$ & $35(32.0-40.7)$ & $27(21.0-30.0)$ & $25.5(20.5-36.2)$ & $35.5(31.2-35.7)$ \\
\hline
\end{tabular}


Table 2 Relative abundance of the 6 dominant phyla observed in the upper and lower respiratory tract of healthy horses $(n=6)$ at day 0 , and relative abundance of genus within each phylum

Phylum (mean relative abundance, \%) Genus (mean relative abundance

$\begin{array}{ll}\text { Proteobacteria (43.85\%) } & \text { Sphingomonas (35.69\%) }\end{array}$

$\begin{array}{ll}\text { Proteobacteria (43.85\%) } & \text { Sphingomonas (35.69\%) } \\ & \text { Pantoea (26.65\%) } \\ & \text { Pseudomonas (14.57\%) } \\ & \text { Massilia (5.59\%) } \\ & \text { Rhizobium (3.35\%) } \\ & \text { Mesorhizobium (2.20\%) } \\ & \text { Naxibacter (1.60\%) }\end{array}$

Serratia (1.39\%)

Devosia (1.32\%)

Actinobacteria (21.63\%)

Finnicutes (16.82\%)

Bacteroidetes (13.24\%)

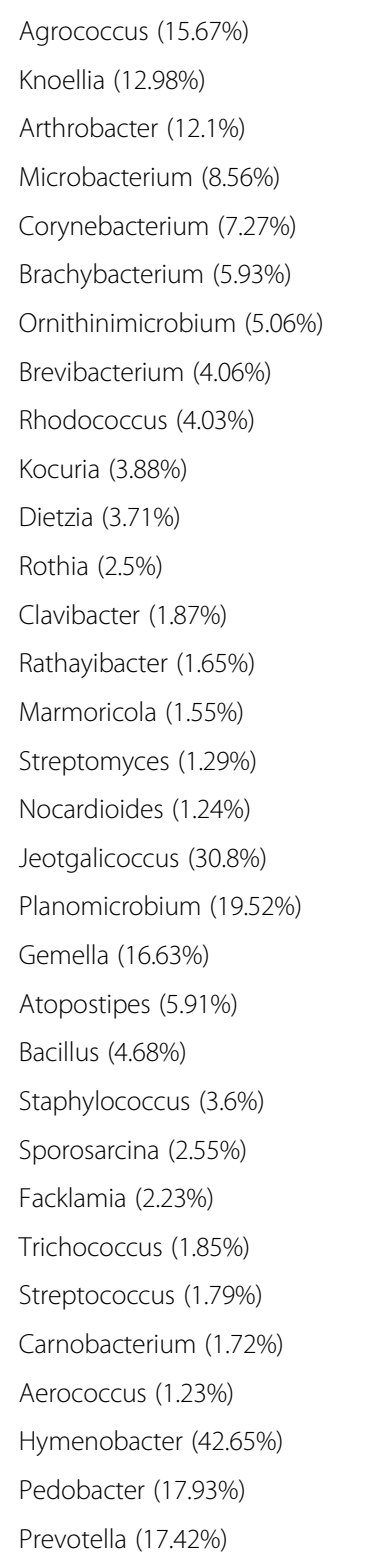

Table 2 Relative abundance of the 6 dominant phyla observed in the upper and lower respiratory tract of healthy horses $(n=6)$ at day 0 , and relative abundance of genus within each phylum (Continued)

\begin{tabular}{ll}
\hline & Flavisolibacter (12.19\%) \\
& Gillisia (3.1\%) \\
& Chryseobacterium (2.69\%) \\
& Cloacibacterium (1.66\%) \\
& Ferruginibacter (1.51\%) \\
Verrucomicrobia (0.79\%) & Luteolibacter $(20.93 \%)$ \\
& Akkermansia (8.79\%) \\
Chloroflexi (0.51\%) & Sphaerobacter (97.84\%) \\
& Litorilinea (2.16\%) \\
\hline
\end{tabular}

appropriately controls for over-dispersed data and variable library sizes [34]. A $p$-value cut-off of 0.05 was specified for rejecting the null hypothesis of no difference between groups.

\section{Results}

Bronchoalveolar lavages results and enrolment

Bronchoalveolar lavages results are shown in Table 1. Seven horses were enrolled in the IAD group based on BAL cytology. Six horses were classified as healthy based on their BAL cytology; three were randomly enrolled in the DEX group and received dexamethasone for 10 days and three were enrolled in the CONTROL group. There was no significant difference in differential cell count for any cell type in the BAL fluid between day 0 and day 11 (Table 1).

\section{Microbiota overview}

An average of 13,524 sequences per sample (min: 1219; max: 81,289) were obtained after removal of (i) contaminating OTUs identified in the control samples ( $n=20$ OTUs; Additional file 1: Figure S1), and (ii) samples with less than 1000 sequences $(n=1)$. Using de-novo clustering, 2209 OTUs were identified and 963 OTUs remained after filtering low abundance and rare OTUs.

\section{Upper and lower respiratory tract microbiota in healthy horses}

Nineteen phyla were identified in the respiratory tract of healthy horses at day $0(n=6)$, with six phyla showing a relative abundance $>0.1 \%$ : Actinobacteria, Bacteroidetes, Chloroflexi, Firmicutes, Proteobacteria and Verrucomicrobia (Table 2, Fig. 2). Four phyla represented 95.54\% of the total abundance: Proteobacteria (43.85\%), Firmicutes (16.82\%), Bacteroidetes (13.24\%) and Actinobacteria (21.63\%) (Additional file 2: Figure S2). At the genus level, 50 genera had a relative abundance $>0.1 \%$ with 


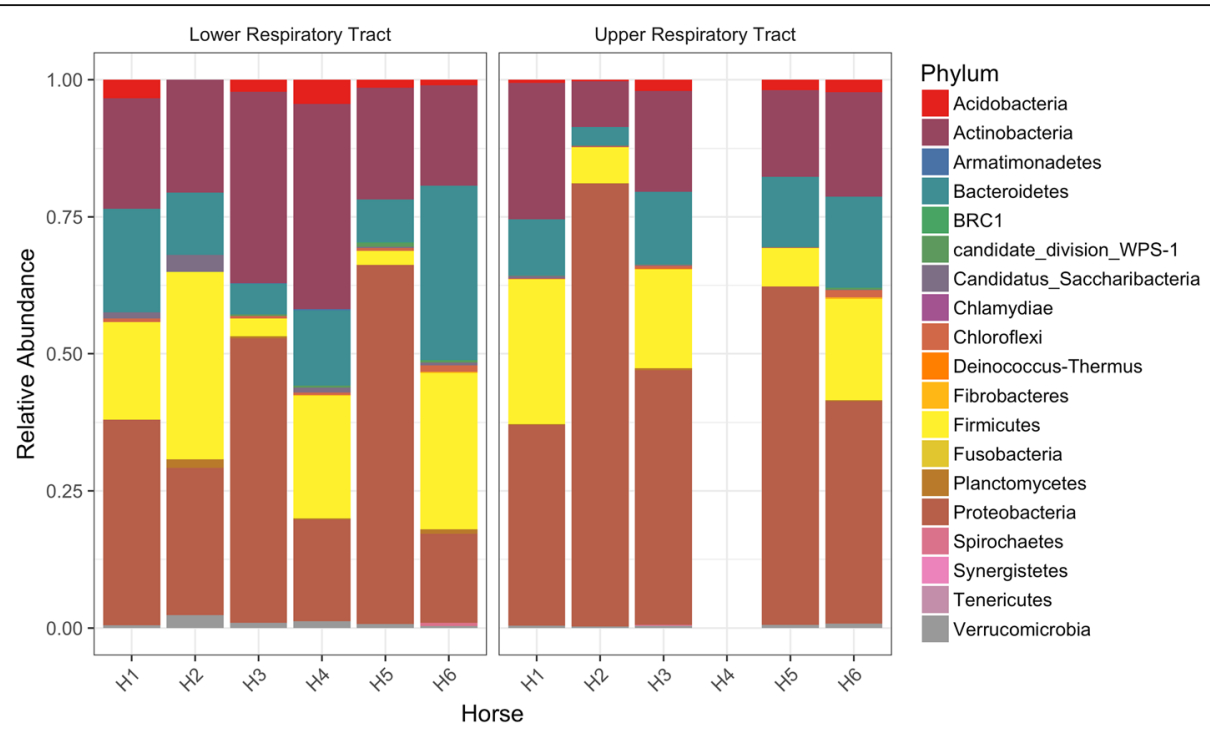

Fig. 2 Phyla relative abundance in the upper and lower respiratory tract in the healthy horses $(n=6 ; \mathrm{H} 1$ to $\mathrm{H6}$ ). Note that the upper respiratory tract sample for horse $4(\mathrm{H} 4)$ had low numbers of sequences after filtering contaminants and was discarded

Sphingomonas and Pantoea being the most abundant (Table 2).

Nonmetric multidimensional scaling (NMDS) ordination with Bray-Curtis distance showed differences in bacterial communities between the upper and lower respiratory tract of healthy horses (Fig. 3a). Testing for a difference between the upper and lower respiratory tract microbiota revealed a change in the relative abundance of 2 OTUs, with Moraxella increased in the upper respiratory tract, and Cupriavidus increased in the lower respiratory tract (Additional file 3: Figure S3). There was also a significant decrease in richness at the lower respiratory tract level based on Chao1 $(p=0.0043$, Wilcoxon test) (Fig. 3b). However, Shannon index did not differ between the upper and lower respiratory tract $(p=0.93$, Wilcoxon test) (Fig. 3b), indicating that while there was an overall decrease in species richness, evenness remained unchanged.

\section{Differences between healthy and IAD horses}

Differences in bacterial communities at the upper and lower respiratory tract level between disease status were visualized using NMDS (Fig. 4). Between healthy and IAD horses, 6 OTUs (Fig. 5) differed in the lower airways based on the generalized linear model. In horses with IAD, relative abundance of Streptococcus and an OTU assigned to the phylum Candidatus_Saccharibacteria were increased, whereas relative abundance of Psychrobacter, Rhodococcus, Aerococcus and Hymenobacter spp. were decreased (Fig. 5). No differentially abundant OTUs were identified between disease status in the upper respiratory tract.

\section{Effects of dexamethasone on the upper and lower respiratory tract microbiota}

Dexamethasone had an effect on the lower respiratory tract microbiota of both healthy and IAD horses (Fig. 6); the treatment effect was not different between disease status. After 10 days of dexamethasone administration, the lower respiratory tract of both healthy and IAD horses experienced a significant change in the abundance of 11 OTUs (Additional file 4: Figure S4), with 9 OTUs responding similarly between disease status (Fig. 7). Peptostreptococcus, Porphyromonas, Filifactor, Streptococcus, Porphyromonas, Parvimonas, Fusobacterium and Bacteroides spp. increased from day 0 to day 11 (pre vs post treatment), whereas Candidatus_Saccharibacteria OTU decreased. There was evidence that dexamethasone treatment also decreased evenness in the lower airways of both healthy and IAD horses, however, this decrease was not statistically significant based on our pre-set level of significance (Additional file 5: Figure S5). No treatment effect was observed on the upper respiratory tract microbiota (Fig. 6).

No differentially abundant OTUs were detected between time points ( $\mathrm{d} 0$ and $\mathrm{d} 11$ ) for the CONTROL group (i.e. healthy horses not treated with dexamethasone; data not shown) indicating that time alone did not significantly influence the airways microbiota.

\section{Discussion}

This study investigated for the first time the differences in airway community profiles between healthy horses and horses with mild equine asthma (IAD), and the evolution of these communities after dexamethasone 


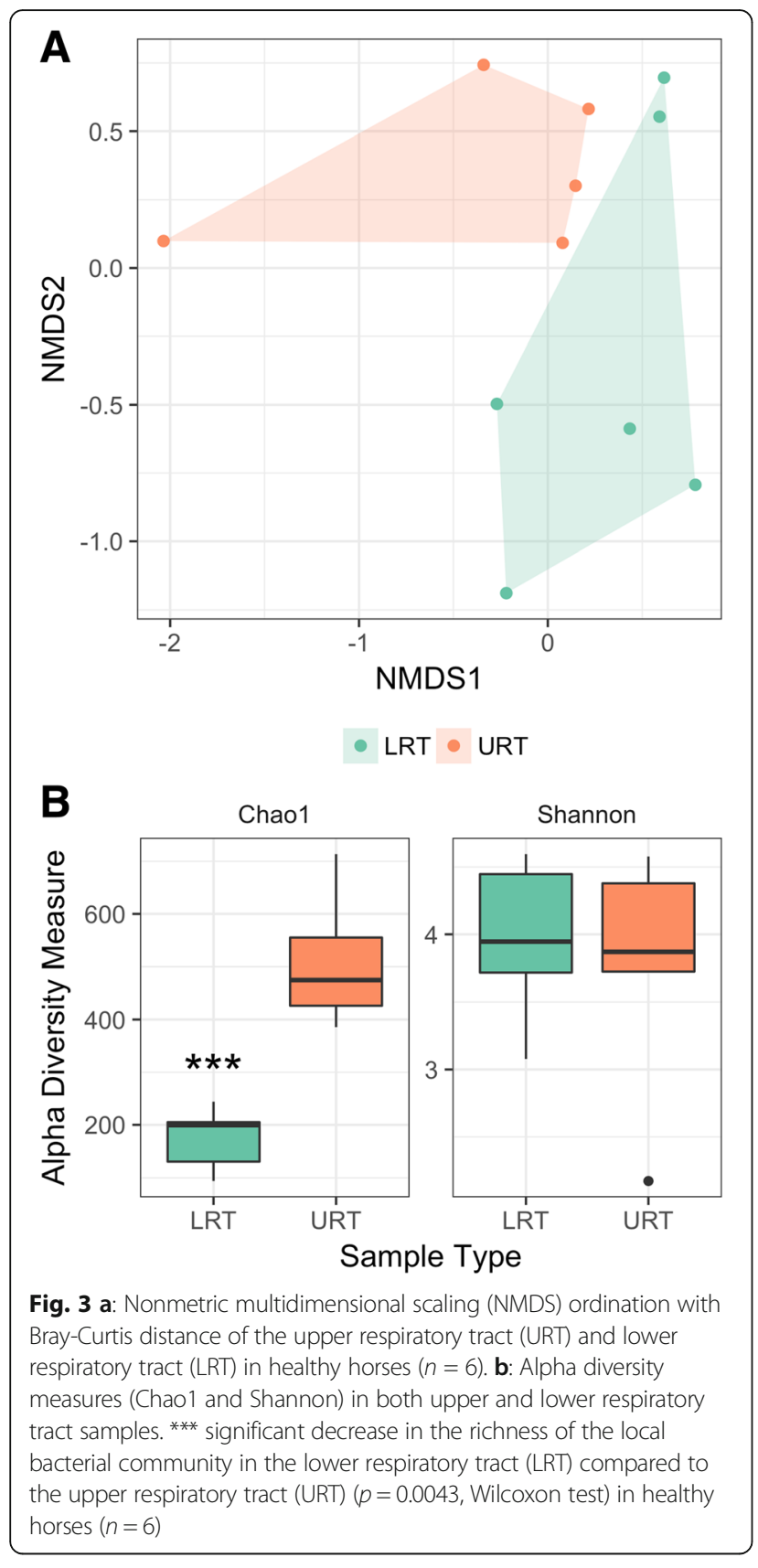

treatment. Using high throughput sequencing, we showed a difference in the lower respiratory tract between healthy and IAD horses, with 6 OTUs having different abundances. However, no differences were observed between disease status at the upper respiratory tract level. Dexamethasone induced a distinct shift in the community structure of the lower respiratory tract in both healthy horses and those with IAD, with a significant change in the abundance of 11 OTUs, with 9 OTUs responding similarly to treatment. A difference in community structure was also observed between the upper and lower respiratory tract of healthy horses.
Strengths of our study design include strict attention to controls, with the inclusion of negative controls at sample collection, during extraction, and throughout sequencing. This resulted in the removal of 20 potential contaminants from analysis. Previous reports warned that contamination is a critically important issue in sequence-based microbiome analysis, particularly when samples contain a low biomass $[35,36]$. Another strength of our study was the technique used to collect the lower respiratory tract samples, the percutaneous transtracheal method, which is superior at avoiding contamination than using a sheathed catheter through an endoscope [18, 37]. The OTUs filtering protocol used was also stringent, which limited the impact of contamination on results presented. Finally, all horses resided on the same property and paddock, which controlled for the potential confounding variables such as location, environmental management, and husbandry procedures. A limitation of this study is its small sample size $(n=13)$, which could have impaired our ability to detect differences in community profiles and/or OTUs between treatment groups (type 2 error). While we did not observe any apparent outliers in the studied population, the results presented in this manuscript must be interpreted with consideration of the small sample size; results might not generalise well to other equine communities.

The differences observed between healthy and IAD horses at the lower respiratory tract level concurred with the previous report indicating that bacteria could play a role in the pathogenesis of mild equine asthma [9-11]. However, in the present study, only the abundance of Streptococcus spp. was increased in horses with IAD and we did not observe a significant increase in Actinobacillus spp., Acinetobacter spp. and Mycoplasma spp. as reported previously [9]. Interestingly, the presence of Streptococcus (S. zooepidemicus and S. pneumonia) in tracheal washes were previously associated with lower airway inflammation in a study conducted on 278 Thoroughbred racehorses [38]. Furthermore, a study on human asthma also reported that the genera with the highest relative abundance in bronchial biopsy samples was Streptococcus [39]. Based on this finding, it seems that further research is warranted on the role of Streptococcus on asthma pathogenesis in horses.

As expected, dexamethasone had a significant treatment effect on the microbiota of the lower respiratory tract in horses. After dexamethasone treatment, numerous OTUs increased in abundance, including Streptococcus spp., while Candidatus_Saccaribacteria OTU decreased in abundance. This overgrowth of certain bacteria in the lower respiratory tract could be secondary to the immunomodulation induced by the dexamethasone treatment, with the reduction in Candidatus_Saccaribacteria OTU providing supportive evidence that dexamethasone treatment also decreased evenness in the lower airways of both healthy 


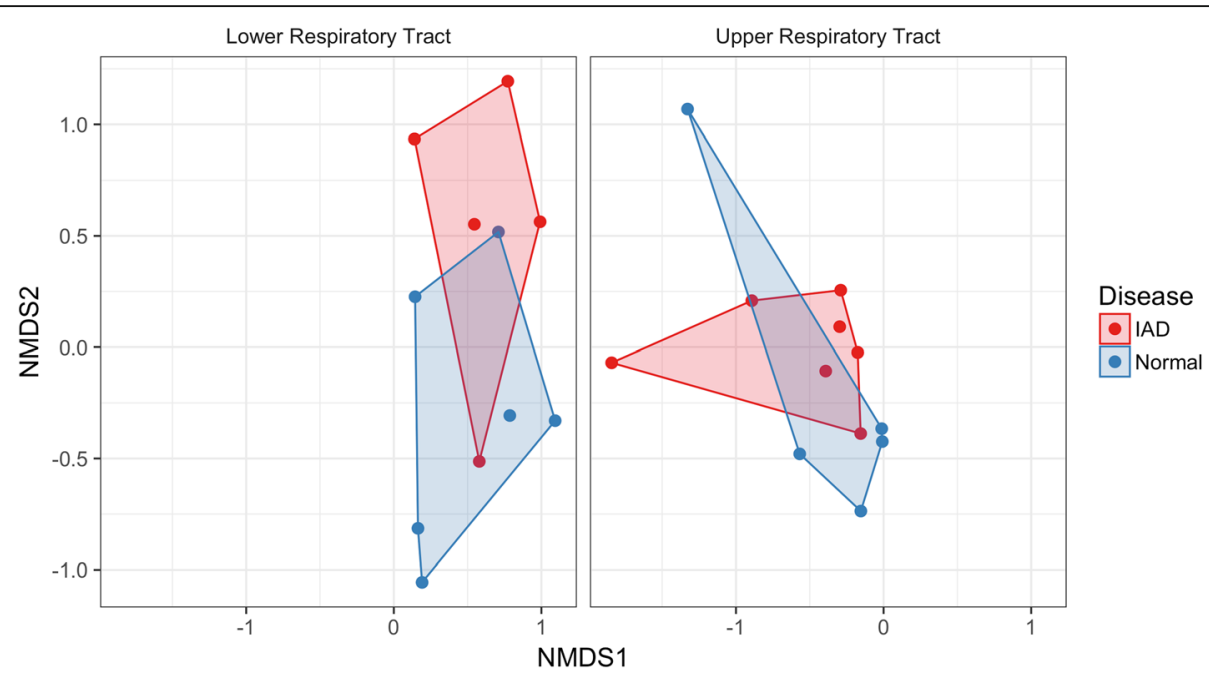

Fig. 4 Nonmetric multidimensional scaling (NMDS) ordination with Bray-Curtis distance of the differences in bacterial communities at the upper respiratory tract and lower respiratory tract level between disease status (Inflammatory Airway Disease [IAD], $n=7 ;$ Normal, $n=6$ )

and IAD horses. In humans, macrolide antibiotic administration has been used in addition to bronchodilators and corticosteroids to improve lung function in asthmatic patients [40]. Perhaps bacterial overgrowth in IAD horses treated with dexamethasone should be controlled to improve treatment success, especially in cases with a poor response to corticosteroids therapy.

The treatment effect of dexamethasone was not different between healthy horses and those with IAD, indicating either (i) that inflammation present in the lower respiratory tract does not influence the immunosuppressive effects of dexamethasone, (ii) that dexamethasone has a stronger effect than the disease on the microbiota, or (iii) that we did not have enough power to detect a difference. We did not include a control group of untreated horses with IAD for ethical reasons as they were privately owned horses; however, there were no OTUs that differed in abundance over the course of the trial in the CONTROL

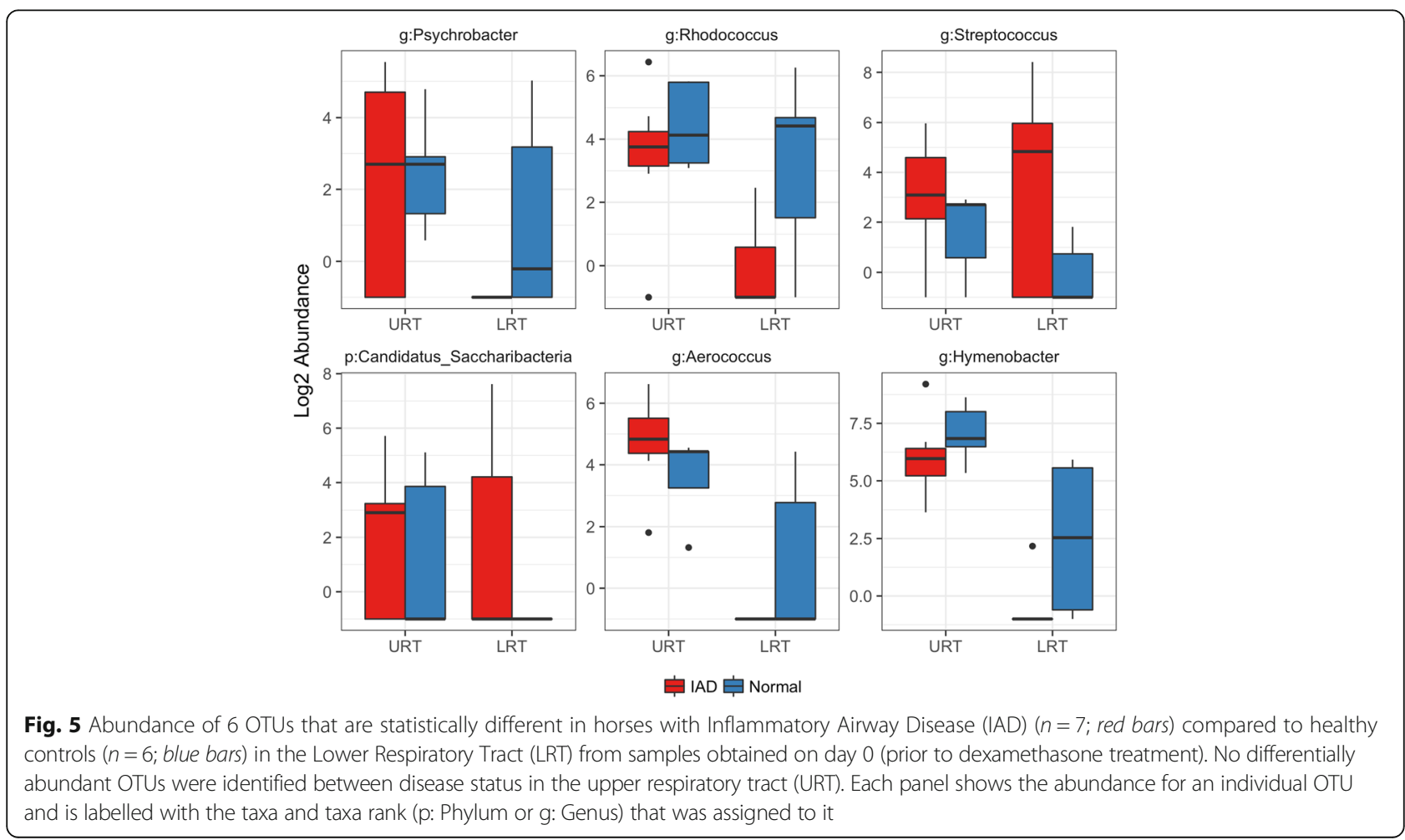




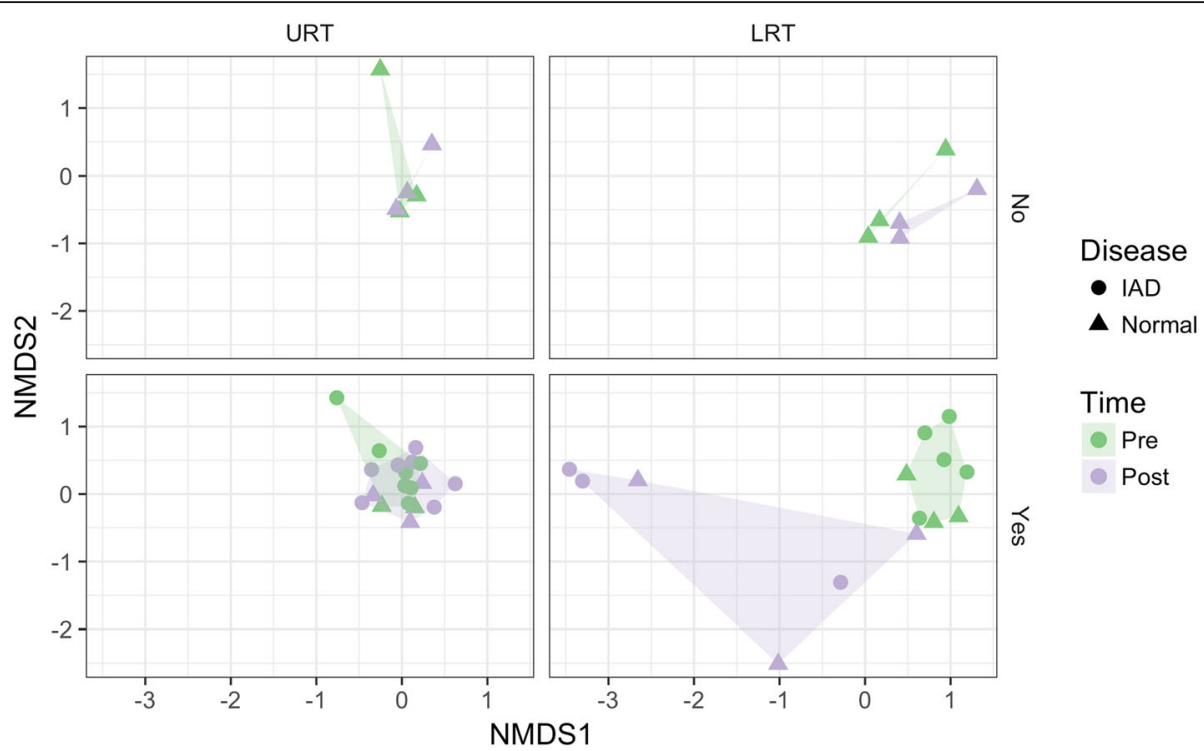

Fig. 6 Nonmetric multidimensional scaling (NMDS) ordination with Bray-Curtis distance of the dexamethasone treatment effect on the lower respiratory tract microbiota of both healthy (Normal; $n=6$; Triangles) and Inflammatory Airway Disease (IAD; $n=7$; Circles) horses ("No": indicates no treatment: $n=3$ Normal horses. "Yes": indicates dexamethasone treatment for 10 days: $n=3$ Normal horses and $n=7$ IAD horses). The horizontal axis is the Upper Respiratory Tract (URT) and Lower Respiratory Tract (LRT), and the vertical axis is dexamethasone treatment (CONTROL group did not receive dexamethasone). Pre and Post indicate day 0 and day 11 sampling time-points, respectively

group, thus indicating that the difference observed in the groups treated with dexamethasone was due to the treatment. While dexamethasone administration has an effect on the lower respiratory tract microbiota, it is interesting to note its lack of effect on BAL cytology in the present study. This finding is consistent with other equine asthma studies, performed on horses with both mild and severe asthma, where an improvement in pulmonary function and clinical signs was not associated with a concurrent decrease in inflammatory cells in BAL $[15,41,42]$.

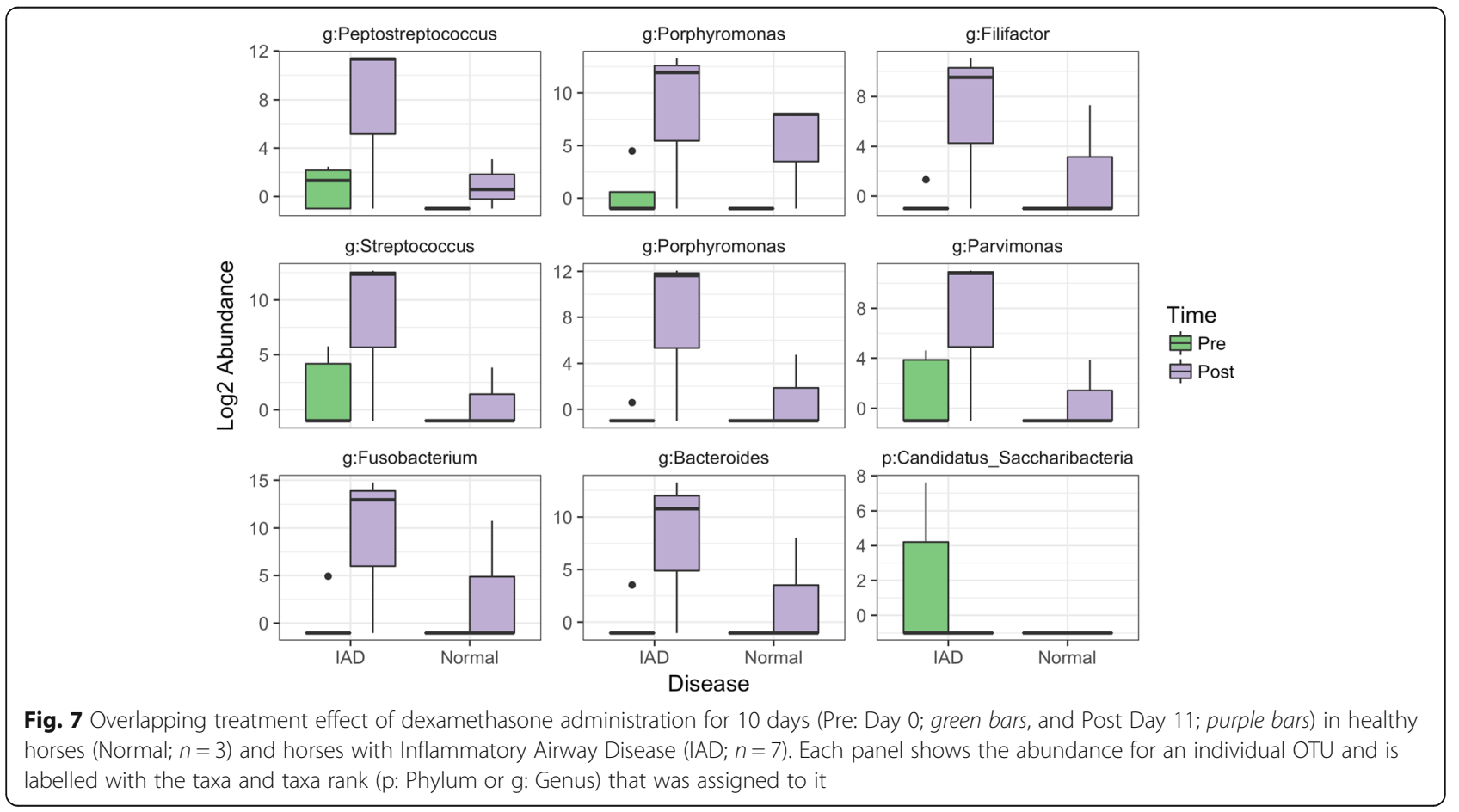


This study demonstrates that the equine lung is not sterile, with the lower respiratory tract possessing a unique microbiota. However, only 2 OTUs differed between upper and lower airways, indicating that the majority of OTUs were overlapping. Interestingly, in healthy humans, the nasal microbiota contributed very little to the composition of the local bacterial communities in the lung, however, there was an overlap with the communities observed in the mouth [43]. That numerous OTUs are shared by the upper and lower tract in the present study can be explained by the fact that horses are obligate nasal breathers and have complete separation of the nasopharynx and oral cavity, except when swallowing, due to an elongated soft palate.

\section{Conclusions}

The respiratory microbiome of horses is diverse, but dominated by four phyla, Proteobacteria, Firmicutes, Bacteroidetes and Actinobacteria. There was a clear separation between the bacterial community in the lower respiratory tract of healthy and IAD horses, with 6 OTUs in the tracheal community having different abundance with disease status, including an increase in Streptococcus in horses with IAD. Based on this finding, further research is warranted on the role of Streptococcus on IAD pathogenesis in horses. Treatment with dexamethasone has a significant treatment effect on the lower respiratory tract microbiome in all horses, with numerous OTUs increasing in abundance, including Streptococcus spp. Perhaps control of bacterial overgrowth in IAD horses treated with dexamethasone could be part of the treatment strategy.

\section{Additional files}

Additional file 1: Figure S1. Abundance of contaminating OTUS identified in the blank samples $(n=20)$. Blank negative controls did not include water (kit only). (TIFF 21097 kb)

Additional file 2: Figure S2. Top 10 genera (by relative abundance) in each of the major phyla identified in the upper and lower respiratory tract of 6 healthy horses ( $\mathrm{H} 1 \mathrm{to} \mathrm{H6}$ ). (TIFF $35863 \mathrm{~kb}$ )

Additional file 3: Figure S3. Abundance of 2 OTUs (labelled with genus) that differed between the upper respiratory tract (URT) and lower respiratory tract (LRT) of healthy horses $(n=6)$. (TIFF $14065 \mathrm{~kb})$

Additional file 4: Figure S4. Dexamethasone treatment (10 days) effect (Pre: day 0; green bars and Post: day 11; purple bars) in the lower respiratory tract of both healthy $(n=6)$ and Inflammatory Airway Disease (IAD, $n=7)$ horses. Each panel shows the abundance for an individual OTU and is labelled with the taxa and taxa rank (p: Phylum or g: Genus) that was assigned to it. (TIFF $42192 \mathrm{~kb}$ )

Additional file 5: Figure S5. Alpha diversity measures (Chao1 and Shannon) of dexamethasone treatment (10 days) effect (Pre: day 0; green bars and Post: day 11; purple bars) in both upper respiratory tract and lower respiratory tract samples. There was no significant decrease in the evenness in the lower airways of both healthy (Normal) and Inflammatory Airway Disease (IAD) horses after $p$-value adjustment for multiple comparisons ( $p=0.071$, Wilcoxon test). (TIFF $8440 \mathrm{~kb}$ )

\section{Abbreviations}

BAL: Bronchoalveolar Lavage; CONTROL: Healthy control group (no dexamethasone) $(n=3)$; DEX: Healthy dexamethasone group $(n=3)$; IAD: Inflammatory Airway Disease (mild equine asthma); LRT: Lower Respiratory Tract; NMDS: Nonmetric Multidimensional Scaling; NPS: Nasopharyngeal Swab; OTU: Operational Taxonomic Unit; TTW: Transtracheal Wash; URT: Upper Respiratory Tract

\section{Acknowledgements}

The authors would like to acknowledge Persephone Greco-Otto, for help with sample collection; Long Jin, for assistance with DNA extraction; and Shelly Wegener, for performing the library preparation and 165 sequencing

\section{Funding}

Internal "Eyes High" funding from the University of Calgary, Faculty of Veterinary medicine.

\section{Availability of data and materials}

The datasets used and/or analysed during the current study are available from the corresponding author on reasonable request. Most of the data is included in this published article [and its Additional files 1, 2, 3, 4 and 5].

\section{Authors' contributions}

SB carried out the study design, sample collection, sample processing and extraction, statistical analysis and drafted the manuscript. ET conceived the study and participated in its design, coordination of materials for sample collection and helped to draft the manuscript. MW performed the OTU table construction, diversity analysis and helped to draft the manuscript. TA participated in the DNA extraction. RL participated in study design, sample collection, BALF analysis and group allocation, and helped to draft the manuscript. All authors read and approved the final manuscript.

\section{Ethics approval and consent to participate}

This study was conducted in strict accordance with the recommendations of the Canadian Council of Animal Care.

This research protocol was reviewed and approved by the University of Calgary Veterinary Sciences Animal Care Committee (ACC17-0036) and a consent form was signed by the owner of the horses.

Consent for publication

Not applicable.

Competing interests

The authors declare they have no competing interests.

\section{Publisher's Note}

Springer Nature remains neutral with regard to jurisdictional claims in published maps and institutional affiliations.

\section{Author details}

${ }^{1}$ Faculty of Veterinary Medicine, University of Calgary, Calgary, AB, Canada. ${ }^{2}$ Lethbridge Research Centre, Agriculture and Agri-Food Canada, Lethbridge, $A B$, Canada.

Received: 1 March 2017 Accepted: 14 August 2017

Published online: 23 August 2017

\section{References}

1. Robinson NE. International workshop on equine chronic airway disease. Michigan State University 16-18 June 2000. Equine Vet J. 2001;33(1):5-19.

2. Couetil LL, Hoffman AM, Hodgson J, Buechner-Maxwell V, Viel L, Wood JL, Lavoie JP. Inflammatory airway disease of horses. J Vet Intern Med. 2007;21(2):356-61.

3. Leguillette R. Recurrent airway obstruction - heaves. Vet Clin North Am Equine Pract. 2003;19(1):63-86. vi

4. Wasko AJ, Barkema HW, Nicol J, Fernandez N, Logie N, Leguillette R. Evaluation of a risk-screening questionnaire to detect equine lung inflammation: results of a large field study. Equine Vet J. 2011;43(2):145-52.

5. Bedenice D, Mazan MR, Hoffman AM. Association between cough and cytology of bronchoalveolar lavage fluid and pulmonary function in horses diagnosed with inflammatory airway disease. J Vet Intern Med. 2008;22(4): 1022-8. 
6. Couetil LL, Rosenthal FS, DeNicola DB, Chilcoat CD. Clinical signs, evaluation of bronchoalveolar lavage fluid, and assessment of pulmonary function in horses with inflammatory respiratory disease. Am J Vet Res. 2001;62(4):538-46.

7. Richard EA, Fortier GD, Denoix JM, Art T, Lekeux PM, Van Erck E. Influence of subclinical inflammatory airway disease on equine respiratory function evaluated by impulse oscillometry. Equine Vet J. 2009;41(4):384-9.

8. Couetil LL, Cardwell JM, Gerber V, Lavoie JP, Leguillette R, Richard EA. Inflammatory airway disease of horses-revised consensus statement. J Vet Intern Med. 2016;30(2):503-15.

9. Wood JL, Newton JR, Chanter N, Mumford JA. Association between respiratory disease and bacterial and viral infections in British racehorses. $J$ Clin Microbiol. 2005;43(1):120-6.

10. Burrell MH, Wood JL, Whitwell KE, Chanter N, Mackintosh ME, Mumford JA Respiratory disease in thoroughbred horses in training: the relationships between disease and viruses, bacteria and environment. Vet Rec. 1996; 139(13):308-13.

11. Christley RM, Hodgson DR, Rose RJ, Wood JL, Reid SW, Whitear KG, Hodgson JL. A case-control study of respiratory disease in thoroughbred racehorses in Sydney. Australia Equine Vet J. 2001;33(3):256-64.

12. Huang YJ, Nelson CE, Brodie EL, Desantis TZ, Baek MS, Liu J, Woyke T, Allgaier M, Bristow J, Wiener-Kronish JP, et al. Airway microbiota and bronchial hyperresponsiveness in patients with suboptimally controlled asthma. J Allergy Clin Immunol. 2011;127(2):372-81. e371-373

13. Hilty M, Burke C, Pedro H, Cardenas P, Bush A, Bossley C, Davies J, Ervine A, Poulter L, Pachter L, et al. Disordered microbial communities in asthmatic airways. PLoS One. 2010;5(1):e8578.

14. Klepac-Ceraj V, Lemon KP, Martin TR, Allgaier M, Kembel SW, Knapp AA, Lory S, Brodie EL, Lynch SV, Bohannan BJ, et al. Relationship between cystic fibrosis respiratory tract bacterial communities and age, genotype, antibiotics and Pseudomonas Aeruginosa. Environ Microbiol. 2010;12(5): 1293-303.

15. Léguillette R., Tohver T., Bond S., Nicol J., McDonald, K.: Effect of dexamethasone and fluticasone on airway hyperresponsiveness in horses with inflammatory airway disease (IAD). J Vet Int Med 2017, Online ahead of print publication.

16. Cohn LA. The influence of corticosteroids on host defense mechanisms. J Vet Intern Med. 1991;5(2):95-104.

17. Pusterla N, Mapes S, Wilson WD. Diagnostic sensitivity of nasopharyngeal and nasal swabs for the molecular detection of EHV-1. Vet Rec. 2008; 162(16):520-1.

18. Christley RM, Hodgson DR, Rose RJ, Reid SW, Hodgson JL. Comparison of bacteriology and cytology of tracheal fluid samples collected by percutaneous transtracheal aspiration or via an endoscope using a plugged, guarded catheter. Equine Vet J. 1999;31(3):197-202.

19. Stone B, G R. Cytology sample collection and preparation for veterinary practitioners. Sydney: Vetnostics QML Pathology; 2010.

20. Martin M. Cutadapt removes adapter sequences from high-throughput sequencing reads. EMBnet journal. 2011;17(1):10-2

21. Edgar RC. UPARSE: highly accurate OTU sequences from microbial amplicon reads. Nat Methods. 2013;10(10):996-8.

22. Edgar RC, Flyvbjerg H. Error filtering, pair assembly and error correction for next-generation sequencing reads. Bioinformatics. 2015;31(21):3476-82.

23. Wang Q, Garrity GM, Tiedje JM, Cole JR. Naive Bayesian classifier for rapid assignment of rRNA sequences into the new bacterial taxonomy. Appl Environ Microbiol. 2007;73(16):5261-7.

24. Callahan BJ, McMurdie PJ, Rosen MJ, Han AW, Johnson AJ, Holmes SP. DADA2: high-resolution sample inference from Illumina amplicon data. Nat Methods. 2016;13(7):581-3.

25. Nawrocki EP. Structural RNA homology search and alignment using covariance models. St. Louis: Washington University; 2009.

26. Koster J, Rahmann S. Snakemake-a scalable bioinformatics workflow engine. Bioinformatics. 2012;28(19):2520-2.

27. R Core Team. R: A language and environment for statistical computing. Vienna: R Foundation for Statistical Computing; 2014.http://www.R-project.org/.

28. McMurdie PJ. Holmes S: phyloseq: an R package for reproducible interactive analysis and graphics of microbiome census data. PLoS One. 2013;8(4): e61217.

29. Oksanen J BF, Kindt R, Legendre P, Minchin PR, O'Hara RB, Simpson GL, Solymos P, Stevens MH, Szoecs E, H W: "package vegan": community ecology package. In. https://cran.r-project.org: CRAN; 2015.
30. Shannon CE. A mathematical theory of communication. Bell Syst Tech J. 1948;27(3):379-423.

31. Chao A. Estimating the population size for capture-recapture data with unequal catchability. Biometrics. 1987;43(4):783-91.

32. Benjamini $Y$, Hochberg Y. Controlling the false discovery rate: a practical and powerful approach to multiple testing. Journal of the Royal Statistical Society Series. 1995;57:289-300.

33. Love Ml, Huber W, Anders S. Moderated estimation of fold change and dispersion for RNA-seq data with DESeq2. Genome Biol. 2014;15(12):550.

34. McMurdie PJ, Holmes S. Waste not, want not: why rarefying microbiome data is inadmissible. PLoS Comput Biol. 2014;10(4):e1003531.

35. Salter SJ, Cox MJ, Turek EM, Calus ST, Cookson WO, Moffatt MF, Turner P, Parkhill J, Loman NJ, Walker AW. Reagent and laboratory contamination can critically impact sequence-based microbiome analyses. BMC Biol. 2014;12:87.

36. Weiss S, Amir A, Hyde ER, Metcalf JL, Song SJ, Knight R. Tracking down the sources of experimental contamination in microbiome studies. Genome Biol. 2014;15(12):564.

37. Sweeney CR, Sweeney RW 3rd, Benson CE. Comparison of bacteria isolated from specimens obtained by use of endoscopic guarded tracheal swabbing and percutaneous tracheal aspiration in horses. J Am Vet Med Assoc. 1989; 195(9):1225-9.

38. Wood JL, Burrell MH, Roberts CA, Chanter N, Shaw Y. Streptococci and Pasteurella spp. associated with disease of the equine lower respiratory tract. Equine Vet J. 1993:25(4):314-8.

39. Millares L, Bermudo G, Perez-Brocal V, Domingo C, Garcia-Nunez M, Pomares X, Moya A, Monso E. The respiratory microbiome in bronchial mucosa and secretions from severe lgE-mediated asthma patients. BMC Microbiol. 2017;17(1):20.

40. Kostadima E, Tsiodras S, Alexopoulos El, Kaditis AG, Mavrou I, Georgatou N, Papamichalopoulos A. Clarithromycin reduces the severity of bronchial hyperresponsiveness in patients with asthma. Eur Respir J. 2004;23(5):714-7.

41. Robinson NE, Berney C, Behan A, Derksen FJ. Fluticasone propionate aerosol is more effective for prevention than treatment of recurrent airway obstruction. J Vet Intern Med. 2009;23(6):1247-53.

42. Lavoie JP, Leguillette R, Pasloske K, Charette L, Sawyer N, Guay D, Murphy T, Hickey GJ. Comparison of effects of dexamethasone and the leukotriene D4 receptor antagonist L-708,738 on lung function and airway cytologic findings in horses with recurrent airway obstruction. Am J Vet Res. 2002; 63(4):579-85.

43. Bassis CM, Erb-Downward JR, Dickson RP, Freeman CM, Schmidt TM, Young VB, Beck JM, Curtis JL, Huffnagle GB. Analysis of the upper respiratory tract microbiotas as the source of the lung and gastric microbiotas in healthy individuals. MBio. 2015;6(2):e00037.

\section{Submit your next manuscript to BioMed Central and we will help you at every step:}

- We accept pre-submission inquiries

- Our selector tool helps you to find the most relevant journal

- We provide round the clock customer support

- Convenient online submission

- Thorough peer review

- Inclusion in PubMed and all major indexing services

- Maximum visibility for your research

Submit your manuscript at www.biomedcentral.com/submit 